\title{
A Symmetric Numerical Range for Matrices
}

\author{
B. David Saunders and Hans Schneider*
}

Received October 2, 1975

Summary. For each norm $y$ on $\mathbb{C}^{n}$, we define a numerical range $Z_{v}$, which is symmetric in the sense that $Z_{v}=Z_{\nu D}$, where $\nu^{D}$ is the dual norm.

We prove that, for $a \in \mathbb{C}^{n n}, Z_{v}(a)$ contains the classical field of values $V(a)$. In the special case that $\nu$ is the $l_{1}$-norm, $Z_{\nu}(a)$ is contained in a set $G(a)$ of Gershgorin type defined by C. R. Johnson.

When $a$ is in the complex linear span of both the Hermitians and the $\nu$-Hermitians, then $Z_{v}(a), V(a)$ and the convex hull of the usual $\nu$-numerical range $V_{v}(a)$ all coincide. We prove some results concerning points of $V(a)$ which are extreme points of $Z_{y}(a)$.

\section{Introduction}

In this note we introduce a numerical range $Z_{y}$ for matrices, where $v$ is a norm on $\mathbb{C}^{n}$. We call this numerical range symmetric since $Z_{\nu}=Z_{\nu}$, where $\nu^{D}$ defined by $\nu^{D}(y)=\sup \left\{\left|y^{*} x\right| / p(x): 0 \neq x \in \mathbb{C}^{n}\right\}$ is the dual norm of $\nu$. For $a \in \mathbb{C}^{n n}$, we compare $Z_{\nu}(a)$ with numerical ranges already in the literature:

(1.1) The classical field of values (Hausdorff [8], Toeplitz [13]) is

$$
V(a)=\left\{\frac{x^{*} a x}{x^{*} x}: 0 \neq x \in \mathbb{C}^{n}\right\} \text {. }
$$

(1.2) The generalization of $V(a)$ introduced by Bauer [1] is

$$
V_{\nu}(a)=\left\{y^{*} a x:(x, y) \in \Pi_{v}\right\},
$$

where $\nu$ is a norm on $\mathbb{C}^{n}$ and

$$
\Pi_{v}=\left\{(x, y) \in \mathbb{C}^{n} \times \mathbb{C}^{n}: \nu(x)=v^{D}(y)=y^{*} x=1\right\} .
$$

For variants of $\Pi_{y}$, see Lumer [10], Bonsall-Duncan [3], Bauer [2] and DeutschZenger [5].

(1.3) The convex hull of the "mixed" Gershgorin circles introduced by C. R. Johnson [9] is

$$
G(a)=\operatorname{conv} \bigcup_{j=1}^{n} G_{j}(a),
$$

* Part of this research was done while the authors were at the Mathematische Institut, Technische Universität, München, West Germany. The first author presented these results at the Seminar on Matrix Theory (Positivity and Norms) held in Munich in December 1974. The second author also acknowledges support from the National Science Foundation under grant GP 37978X 
where conv stands for convex hull and for $j=1, \ldots, n$

$$
G_{j}(a)=\left\{\xi \in \mathbb{C}:\left|\xi-a_{j j}\right| \leqq \frac{1}{2} \sum_{\substack{i=1 \\ i \neq j}}^{n}\left(\left|a_{i j}\right|+\left|a_{j i}\right|\right)\right\} .
$$

Johnson proved that $V(a) \leq G(a)$, for all $a \in \mathbb{C}^{n n}$. We show that $V(a) \leq Z_{\nu}(a)$, for all norms $\nu$. If $\nu$ is the $l_{1}$-norm (or the $l_{\infty}$-norm) we show that $Z_{v}(a) \leq G(a)$. Further if $a$ is Hermitian then, for all norms $\nu, Z_{\nu}(a) \leq \mathbb{R}$. We show by an example that there exists $a \in \mathbb{C}^{n n}$ and a norm $\nu$ such that $Z_{\nu}(a) \subset V_{v}(a) \cap V_{\nu}(a)$. Thus $Z_{v}$ is of numerical interest. In the special case that $\nu$ is the $l_{1}$-norm, our bounds for $V(a)$ are sharper though less easy to compute in general than Johnson's.

In [16], Zenger gave an axiomatic treatment of eigenvalue inclusion sets. This approach is appropriate here, since $Z_{\nu}, G$, and conv $V_{\nu}$ share basic properties. Thus we begin Section 2 by listing axioms for convex numerical ranges, and then we prove the results outlined above. Section 3 is motivated by the result that for each norm $\nu$ there is a $p$-transform $\nu_{p}$ such that the $\nu_{p}$-Hermitians are Hermitian, see Deutsch-Schneider [6, Proposition (4.1)]. Under the hypothesis that $h$ and $k$ are both $\nu$-Hermitian and Hermitian, we show in Section 3 that $Z_{v}(h+i k)=$ conv $V_{v}(h+i k)=V(h+i \mathrm{k})$. Our theorem then leads us to investigate in Section 4 extreme points $\alpha$ of $Z_{v}(a)$ which also belong to $V(a)$. If $\alpha$ is such a point, and $\Pi_{\nu}$ is the set defined in (1.1), then we show that there exist $(x, y) \in \Pi_{\nu}$ such that

$$
\alpha=x^{*} a x / x^{*} x=y^{*} a y / y^{*} y=\frac{1}{2}\left(y^{*} a x+x^{*} a y\right) \text {. }
$$

This results has an application to eigenvectors of norm-Hermitians.

The chief tool in these investigations is a result due to Cain-Saunders-Schneider [4, Theorem 6] from which it follows that for each $z \in \mathbb{C}^{n}$ there is a positive multiple of $z$ which is of the form $x+y$, where $(x, y) \in \Pi_{y}$.

\section{The Symmetric Numerical Range}

(2.1) Definition. A set valued mapping $W: \mathbb{C}^{n n} \rightarrow \mathscr{P}(\mathbb{C})$ is called a (homogeneous, unital, compact) convex numerical range if

(i) $W(a)$ contains the spectrum of $a$, for all $a \in \mathbb{C}^{n n}$,

(ii) $W(a+b) \leq W(a)+W(b)$, for all $a, b \in \mathbb{C}^{n n}$,

(iii) $W(\lambda a)=\lambda W(a), \quad$ for $\lambda \in \mathbb{C}, a \in \mathbb{C}^{n n}$,

(iv) $W(1)=\{1\}$

(v) $W(a)$ is compact for $a \in \mathbb{C}^{n n}$,

(vi) $W(a)$ is convex for $a \in \mathbb{C}^{n n}$.

Remark. Conditions (ii), (iii) and (iv) of (2.1) imply that

and

$$
W(0)=\{0\},
$$

$$
W(a+\lambda)=W(a)+\lambda, \quad \text { for } \lambda \in \mathbb{C}, a \in \mathbb{C}^{n n} .
$$

Examples of convex numerical ranges are $V, G$, and conv $V_{v}$, as defined in (1.1) $-(1.3)$. 
Definition. Let $\nu$ be a norm. For $a \in \mathbb{C}^{n n}$, let.

$$
Z_{v}(a)=\operatorname{conv}\left\{\frac{1}{2}\left(y^{*} a x+x^{*} a y\right):(x, y) \in \Pi_{\nu}\right\} .
$$

It is easy to see that $Z_{\nu}$ satisfies (ii), (iii), and (iv) of Definition (2.1). The compactness of $Z_{\nu}(a)$ is a consequence of the compactness of $\Pi_{v}$. Clearly $Z_{\nu}(a)$ is convex. That $Z_{\nu}$ satisfies (i) is a consequence of Theorem (2.4) below.

By taking $\delta=0$ in Theorem 6 of [4], we immediately obtain the following lemma.

(2.3) Decomposition Lemma. Let $\nu$ be a norm on $\mathbb{C}^{n}$, and let $z \in \mathbb{C}^{n}$. Then there exist unique $(x, y) \in \Pi_{v}$ and $t>0$ such that $t z=x+y$.

We shall call $(x, y)$ the $\Pi_{y}$-decomposition of the direction $z$.

(2.4) Theorem. Let $\nu$ be a norm. Then, for all $a \in \mathbb{C}^{n n}$,

$$
Z_{\nu}(a) \geq V(a) \text {. }
$$

Proof. Suppose first that $\Pi^{+}$is the closed right-half plane and that $0 \in V(a) \leq \Pi^{+}$. Let $z^{*} a z=0$ where $z \in \mathbb{C}^{n}$ and $z^{*} z=1$, and suppose that $(x, y)$ is the $\Pi_{\gamma}$-decomposition of the direction $z$. Thus, for some $t>0, t z=x+y$ and so

$$
0=t^{2} z^{*} a z=x^{*} a x+y^{*} a y+x^{*} a y+y^{*} a x \text {. }
$$

Since $\operatorname{Re}\left(x^{*} a x+y^{*} a y\right) \geqq 0$ it follows that $\alpha$ defined by $\alpha=\frac{1}{2}\left(x^{*} a y+y^{*} a x\right)$ satisfied $\operatorname{Re} \alpha \leqq 0$. Since $\alpha \in Z_{v}(a)$, we have $Z_{\nu}(a) \cap \Pi^{-} \neq \phi$, where $\Pi^{-}$is the closed left-half plane. Let $S$ be any supporting half-space of $Z_{v}(a)$ in the plane. There exists $\theta, 0 \leqq \theta<2 \pi$, such that $e^{i \theta} S$ is a translate of $\Pi^{+}$. If $\beta$ is a point of $V\left(e^{i \theta} a\right)$ with minimal real part, then, for $b=e^{i \theta} a-\beta$, we have $0 \in V(b) \leq \Pi^{+}$. The previous argument shows that $Z_{\nu}(b)$ contains a point of $\Pi^{-}$and since $e^{i \theta} S-\beta$ is a supporting half-space of $Z_{v}(b)$, we have $\Pi^{+} \leq e^{i \theta} S-\beta$. Thus $V(b) \leq e^{i \theta} S-\beta$. It follows that $V(a) \leq S$. Since $Z_{v}(a)$ is the intersection of its supporting half-spaces, the theorem follows.

(2.5) Corollary. For any norm $\nu, Z_{v}$ is a convex numerical range.

Since $Z_{y}=Z_{y}$ we call $Z_{y}$ the symmetric numerical range. If $\nu$ is the Euclidean norm, then $Z_{v}=Z_{v}=V_{v}=V_{y}=V$. Also, if $h \in \mathbb{C}^{n n}$ is Hermitian, $y^{*} h x=x^{*} h y$, for $(x, y) \in \Pi_{\nu}$, whence $Z_{\nu}(h) \leq \mathbb{R}$.

(2.6) Theorem. Let $\nu$ be the $l_{1}$ or $l_{\infty}$-norm on $\mathbb{C}^{n}$ and let $G$ be defined as in (1.3). Then, for all $a \in \mathbb{C}^{n n}$,

$$
Z_{\nu}(a) \leq G(a) .
$$

Proof. It is enough to prove the result when $\nu$ is the $l_{1}$-norm, since then $\nu^{D}$ is the $l_{\infty}$-norm. So let $(x, y) \in \Pi_{v}$. Suppose

$$
x=\left(r_{1} e^{i \theta_{1}}, \ldots, r_{n} e^{i \theta_{n}}\right)^{t}, \quad y=\left(s_{1} e^{i \phi_{1}}, \ldots, s_{n} e^{i \phi_{n}}\right)^{t},
$$

where $r_{i} \geqq 0, s_{i} \geqq 0, i=1, \ldots, n$, and $0 \leqq \theta_{i}, \phi_{i}<2 \pi$. Then $\sum_{i=1}^{n} r_{i}=1$, and $0 \leqq s_{i} \leqq 1$, $i=1, \ldots, n$. Let $N=\{1, \ldots n\}$ and $E=\left\{i \in N: r_{i}>0\right\}$. Then

$$
\begin{aligned}
\frac{1}{2}\left(y^{*} a x+x^{*} a y\right) & =\frac{1}{2} \sum_{i, j \in N}\left(s_{i} a_{i j} r_{j} e^{i\left(\theta_{j}-\phi i\right)}+r_{j} a_{j i} s_{i} e^{-i\left(\theta_{i}-\phi i\right)}\right) \\
& =\sum_{j \in E} r_{j} \xi_{j},
\end{aligned}
$$

8 Numer. Math., Bd. 26 
where, for $j \in E$,

$$
\begin{aligned}
\xi_{j} & =\frac{1}{2} \sum_{i \in N}\left(s_{i} a_{i j} e^{i\left(\theta_{j}-\phi i\right)}+a_{j i} s_{i} e^{-i\left(\theta_{i}-\phi i\right)}\right) \\
& =a_{j j}+\frac{1}{2} \sum_{i \in N \backslash\{j}\left(s_{i} a_{i j} e^{i\left(\theta_{1}-\phi_{1}\right)}+a_{j i} s_{i} e^{-i\left(\theta_{j}-\phi(j)\right.}\right),
\end{aligned}
$$

since, for $j \in E$, we have $s_{j}=1$ and $\theta_{j}=\phi_{j}$.

Hence

$$
\left|\xi_{j}-a_{i j}\right| \leqq \frac{1}{2} \sum_{i \in N \backslash\{j\}}\left(\left|a_{i j}\right|+\left|a_{i j}\right|\right) .
$$

Since $\sum_{j \in E} r_{j}=1$, it follows that $\frac{1}{2}\left(y^{*} a x+x^{*} a y\right) \in G(a)$.

(2.7) Example. Let

and let $\nu$ be the $l_{1}$ norm. Then

$$
a=\left[\begin{array}{ll}
1 & 2 \\
2 & 4
\end{array}\right]
$$

$$
V(a) \subset Z_{v}(a) \subset V_{v}(a) \cap V_{v}(a) \subset G(a)
$$

where all the containments are strict.

For, $V(a)=[0,5], Z_{v}(a)=[-1,6], G(a)=\operatorname{conv}\left(G_{1}(a), G_{2}(a)\right)$, where $G_{1}(a)$ and $G_{2}(a)$ are the circles of radius 2 with center 1 and 4 respectively. By NirschlSchneider [11], $V_{y}(a) \supseteq G_{i}(a), i=1,2$ and $V_{v}(a)$ is non-convex since the segment $[1+2 i, 4+2 i]$ intersects $V_{\nu}(a)$ in the points $\{1+2 i, 4+2 i\}$ only. We also have $V_{v}(a)=V_{v}(a)$. The last remark is a consequence of the next result whose proof is easy.

(2.8) Lemma. Let $\nu$ be a norm on $\mathbb{C}^{n}$ such that $\nu(x)=\hat{=}(\bar{x})$, for all $x \in \mathbb{C}^{n}$. If $a \in \mathbb{C}^{n n}$ is symmetric (viz. $a=a^{t}$ ) then $V_{v}(a)=V_{v}(a)$.

\section{Comparison Between Numerical Ranges}

(3.1) Definition. Let $\nu$ be a norm on $\mathbb{C}^{n}$. Then

and

$$
H(\nu)=\left\{h \in \mathbb{C}^{n n}: V_{\nu}(h) \leq \mathbb{R}\right\},
$$

$$
J(\nu)=\{h+i k: h, k \in H(\nu)\} .
$$

If $\nu$ is the $l_{2}$-norm, then $H(\nu)$ consists of the set of Hermitian matrices and we shall denote this set by $H$. For general $\nu$, Vidav [14] (cf. Bonsall-Duncan [3, p. 51]) proved that if $h \in H(\nu)$ then $V_{v}(h)=\operatorname{conv}(\operatorname{spec} h)$. This equality motivates the more general assumption of our next lemma.

(3.2) Lemma. Let $W_{1}, W_{2}$ be convex numerical ranges. Let $h, k \in \mathbb{C}^{n n}$ and suppose that for all $\alpha, \beta \in \mathbb{R}$

Then for all $\eta, \zeta \in \mathbb{C}$,

$$
W_{1}(\alpha h+\beta k)=W_{2}(\alpha h+\beta k) \leq \mathbb{R} .
$$

$$
W_{1}(\eta h+\zeta k)=W_{2}(\eta h+\zeta k) .
$$

Proof. Let $a=\eta h+\zeta k$ where $\eta, \zeta \in \mathbb{C}$ and put $\eta=\alpha+i \gamma, \zeta=\beta+i \delta$, where $\alpha, \beta, \gamma, \delta \in \mathbb{R}$. For $k=1,2, W_{k}(a) \leq W_{k}(\alpha h+\beta k)+i W_{k}(\gamma h+\delta k)$, whence Re $W_{k}(a) \leq$ 
$W_{k}(\alpha h+\beta k)$. But also $W_{k}(\alpha h+\beta k) \leq W_{k}(a)-i W_{k}(\gamma h+\delta k)$, whence $\operatorname{Re} W_{k}(a)=$ $W_{k}(\alpha h+\beta k)$. It follows that $\operatorname{Re} W_{1}(a)=\operatorname{Re} W_{2}(a)$.

But the same argument shows that for each $\theta, 0 \leqq \theta<2 I I, \operatorname{Re} W_{1}\left(e^{i \theta} a\right)=$ $\operatorname{Re} W_{2}\left(e^{i \theta} a\right)$. Since $W_{1}(a)$ and $W_{2}(a)$ are convex subsets of the plane, we deduce that $W_{1}(a)=W_{2}(a)$.

If $\nu$ is a norm on $\mathbb{C}^{n}$, and $p \in \mathbb{C}^{\boldsymbol{n} n}$ is non-singular, we define the norm $\nu_{p}$ by $\nu_{p}(x)=\nu(p x)$, for all $x \in \mathbb{C}^{n}$. If $p$ is the Loewner-John matrix for $\nu$ as defined in Deutsch-Schneider [6], then $H\left(\nu_{p}\right) \leq H$, see [6, Proposition 4.1]. This motivates the hypothesis of our next theorem. We first state a simple lemma.

(3.3) Lemma. Let $\nu$ be a norm on $\mathbb{C}^{n}$ and let $h, \mathrm{k} \in H(\nu) \cap H$. If $(x, y) \in \Pi_{\nu}$ then $y^{*}(h+i k) x=x^{*}(h+i k) y$.

Proof. $y^{*}(h+i k) x=y^{*} h x+i y^{*} k x=x^{*} h^{*} y+i x^{*} k^{*} y=x^{*} h y+i x^{*} k y=$ $x^{*}(h+i k) y$.

(3.4) Theorem. Let $\nu$ be a norm on $\mathbb{C}^{\natural n}$ and suppose $h, k \in H(\nu) \cap H$. Then

$$
Z_{v}(h+i k)=\operatorname{conv} V_{v}(h+i k)=V(h+i k) .
$$

Proof. Let $a=h+i k$. By Lemma (3.3), $y^{*} a x=\frac{1}{2}\left(y^{*} a x+x^{*} a y\right)$, for all $(x, y) \in \Pi_{v}$. Hence $Z_{v}(a)=\operatorname{conv} V_{v}(a)$. To prove the second equality, observe that

$$
V_{v}(\alpha h+\beta k)=\operatorname{conv} \operatorname{spec}(\alpha h+\beta k)=V(\alpha h+\beta k) \text {, }
$$

by Vidav's Lemma [14]. Hence by Lemma (3.2), conv $V_{y}(h+i k)=V(h+i k)$.

Remark. A considerably better result holds: $Z_{v}(h+i k)=V_{v}(h+i k)$, under the hypothesis of Theorem (3.4). For, using Theorem (3.4), B. D. Saunders [12] shows that for $h, k \in H(\nu), V_{\nu}(h+i k)$ is itself convex.

Since $V_{v p}\left(p^{-1} a p\right)=V_{v}(a)$ (cf. [11, Lemma 2] it follows by [6, Proposition 4.1] that:

(3.5) Corollary. Let $\nu$ be a norm on $\mathbb{C}^{n}$ and let $p$ be the Loewner-John matrix for $\nu$. If $a \in J(\nu)$, then

$$
Z_{v_{p}}\left(p^{-1} a p\right)=\operatorname{conv} V_{v}(a)=V\left(p^{-1} a p\right) .
$$

Proof. By Theorem (3.4),

$$
Z_{v_{p}}\left(p^{-1} a p\right)=V\left(p^{-1} a p\right)=\operatorname{conv} V_{v_{p}}\left(p^{-1} a p\right)=\operatorname{conv} V_{v}(a) .
$$

\section{Extreme Points}

We next prove a theorem concerning extreme points of $Z_{v}(a)$ which also belong to $V(a)$.

(4.1) Theorem. Let $\nu$ be a norm, let $a \in \mathbb{C}^{n n}$, and let $z$ be such that $z^{*} z=1$ and $z^{*} a z$ is an extreme point of $Z_{y}(a)$. If $(x, y)$ is the $\Pi_{y}$-decomposition of the direction $z$, then

$$
z^{*} a z=\frac{x^{*} a x}{x^{*} z}=\frac{y^{*} a y}{y^{*} y}=\frac{1}{2}\left(y^{*} a x+x^{*} a y\right) \text {. }
$$


Proof. Let $\alpha=z^{*} a z$ and put $b=a-\alpha$. For some $t>0$, we have $t z=x+y$ and so

where

$$
0=t^{2} z^{*} b z=x^{*} x \alpha_{1}+y^{*} y \alpha_{2}+2 \alpha_{3}
$$

and

$$
\alpha_{1}=x^{*} b x / x^{*} x, \quad \alpha_{2}=y^{*} b y / y^{*} y,
$$

$$
\alpha_{3}=\frac{1}{2}\left(y^{*} b x+x^{*} b y\right) .
$$

Then, for $i=1,2,3$, we have by Theorem (2,4) that $\alpha_{i} \in Z_{v}(b)$. Let $s=x^{*} x+y^{*} y+2$. If $r_{1}=s^{-1} x^{*} x, r_{2}=s^{-1} y^{*} y, r_{3}=2 s^{-1}$, then $0=\sum_{i=1}^{3} r_{i} \alpha_{i}$ and $\sum_{i=1}^{3} r_{i}=1, r_{i}>0$ for $i=1,2,3$. Since 0 is an extreme point of $Z_{y}(b)$ it follows that $\alpha_{1}=\alpha_{2}=\alpha_{3}=0$. The result follows.

(4.2) Corollary. Let $\nu$ be a norm on $\mathbb{C}^{n}$, and suppose that $h, k \in H(\nu) \cap H$. Let $a=h+i k$. If $\alpha$ is an extreme point of $\operatorname{conv} V_{y}(a)$ then there exist $(x, y) \in \Pi_{y}$ such that

$$
\alpha=y^{*} a x=\frac{x^{*} a x}{x^{*} x}=\frac{y^{*} a y}{y^{*} y} .
$$

Proof. By Theorem (3.4), $\alpha$ is an extreme point of $Z_{v}(a)$ and $\alpha \in V(a)$. Let $z \in \mathbb{C}^{n}$. such that $z^{*} z=1$ and $z^{*} a z=\alpha$. Since $y^{*} a x=x^{*} a y$, by Lemma (3.3), the result follows from Theorem (4.1).

(4.3) Corollary. Let $\nu$ be a norm on $\mathbb{C}^{n}$ and let $h \in H(\nu) \cap H$. Let $\lambda=\min (\operatorname{spec} h)$ (or $\lambda=\max (\operatorname{spec} h)$ ) and suppose $h z=\lambda z$, where $0 \neq z \in \mathbb{C}^{n}$. If $(x, y)$ is the $\Pi_{y^{-}}$ decomposition of the direction $z$, then $h x=\lambda x$ and $h y=\lambda y$.

Proof. By Corollary (4.2), $x^{*} h x / x^{*} x=y^{*} h y / y^{*} y=\lambda$, it is easy to show that if $x^{*} h x / x^{*} x=\lambda$ then $h x=\lambda x$ ([7, p. 142]). Similarly we may prove that $h y=\lambda y$.

Corollary (4.3) is related to a result proved under more general hypotheses concerning the existence of pairs $(x, y) \in \Pi_{y}$ which are pairs of eigenvectors of a matrix, see Zenger $[15,1.8]$.

\section{References}

1. Bauer, F. L.: On the field of values subordinate to a norm. Numer. Math. 4, 103-113 (1962)

2. Bauer, F. L.: Fields of value and Gershgorin discs. Numer. Math. 12, 91-95 (1968)

3. Bonsall, F. F., Duncan, J.: Numerical ranges of operators on normed spaces and of elements of normed algebras. Lond. Math. Soc. Lecture Note Series, 2, Cambridge: U. Press 1971

4. Cain, B.E., Saunders, B. D., Schneider, H.: On the geometry of dual pairs. Studies App. Math. (to appear)

5. Deutsch, E., Zenger, C.: On Bauer's generalized Gershgorin discs. Numer. Math. 24, 63-70 (1975)

6. Deutsch, E., Schneider, H.: Bounded groups and norm-hermitian matrices. Linear Algebra and Appl. 9, 9-27 (1975)

7. Franklin, J. N.: Matrix Theory. Prentice-Hall 1968

8. Hausdorff, F.: Der Wertvorrat einer Bilinearform. Math. Zeitschrift 3, 314-316 (1919) 
9. Johnson, C.R.: A Gershgorin inclusion set for the field of values of a finite matrix. Proc. Amer. Math. Soc. 41, 57-60 (1973)

10. Lumer, G.: Semi-inner product spaces. Trans. Amer. Math. Soc. 10, 29-43 (1961)

11. Nirschl, N., Schneider, H.: The Bauer field of values of a matrix. Numer. Math. 6, 355-365 (1964)

12. Saunders, B.D.: A condition for the convexity of the norm-numerical range of a matrix. Linear Algebra and Appl. (to appear)

13. Toeplitz, O.: Das algebraische Analogon $z$ u einem Satze von Fejer. Math. Zeitschrift 2, 187-197 (1918)

14. Vidav, I.: Eine metrische Kennzeichnung der selbst-adjungierten Operatoren. Math. Zeit. 66, 185-193 (1956)

15. Zenger, $\mathrm{C}$.: On convexity properties of the Bauer field of values of a matrix. Numer. Math. 12, 96-105 (1968)

16. Zenger, C.: Minimal subadditive inclusion domains for the eigenvalues of matrices. Linear Algebra and Appl. (to appear)

B. David Saunders

Mathematical Sciences Department

Rensselaer Polytechnic Inst.

Troy, NY 12181

U.S.A.
Hans Schneider

Mathematics Department

University of Wisconsin

Madison, WI 53706

U.S.A. 